\title{
Predictive factors of mortality in rheumatoid arthritis-associated interstitial lung disease analysed by modified HRCT classification of idiopathic pulmonary fibrosis according to the 2018 ATS/ERS/ JRS/ALAT criteria
}

\author{
Hideaki Yamakawa ${ }^{1,2}$, Shintaro Sato ${ }^{1}$, Emiri Tsumiyama ${ }^{1,2}$, Tomotaka Nishizawa ${ }^{1}$, Rie Kawabe ${ }^{1}$, \\ Tomohiro Oba ${ }^{1}$, Teppei Kamikawa ${ }^{3}$, Masanobu Horikoshi ${ }^{3}$, Keiichi Akasaka ${ }^{1}$, Masako Amano ${ }^{1}$, \\ Kazuyoshi Kuwano ${ }^{2}$, Hidekazu Matsushima ${ }^{1}$ \\ ${ }^{1}$ Department of Respiratory Medicine, Saitama Red Cross Hospital, Saitama, Japan; ${ }^{2}$ Department of Internal Medicine, Division of Respiratory \\ Medicine, Jikei University School of Medicine, Tokyo, Japan; ${ }^{3}$ Department of Rheumatology, Saitama Red Cross Hospital, Saitama, Japan \\ Contributions: (I) Conception and design: H Yamakawa; (II) Administrative support: S Sato; (III) Provision of study materials or patients: H Yamakawa, \\ T Kamikawa, M Horikoshi, M Amano, H Matsushima; (IV) Collection and assembly of data: H Yamakawa, E Tsumiyama, T Nishizawa, T Oba, K \\ Akasaka; (V) Data analysis and interpretation: H Yamakawa, S Sato, K Kuwano, H Matsushima; (VI) Manuscript writing: All authors; (VII) Final \\ approval of manuscript: All authors. \\ Correspondence to: Hideaki Yamakawa, MD, PhD. Department of Respiratory Medicine, Saitama Red Cross Hospital, 1-5 Shintoshin, Chuo-ku, \\ Saitama 330-8553, Japan. Email: hide1144@jikei.ac.jp.
}

Background: Interstitial lung disease (ILD) is associated with high morbidity and mortality in rheumatoid arthritis (RA). Although usual interstitial pneumonia (UIP) pattern was reported as a poor prognostic factor, in clinical practice, we often cannot classify high-resolution computed tomography (HRCT) patterns specifically as UIP or nonspecific interstitial pneumonia (NSIP). This study of RA-ILD aimed to elucidate prognosis by using our modified HRCT pattern classification according to the latest guideline on idiopathic pulmonary fibrosis (IPF).

Methods: We analysed the medical records of 96 consecutive patients diagnosed as having RA-ILD. The modified HRCT classifications were defined as definite UIP, probable UIP, indeterminate for UIP (i.e., early UIP or NSIP/UIP), NSIP, organizing pneumonia (OP), NSIP+OP, and unclassifiable. Predictors of prognosis were determined using Cox regression models.

Results: Our RA-ILD cohort included definite UIP (21\%), probable UIP (20\%), indeterminate for UIP (30\%) including NSIP/UIP (27\%), alternative diagnosis (29\%) including NSIP (14\%), and other patterns. Interrater agreement for HRCT pattern was good $(\kappa=0.75)$. Multivariate analysis showed that older age, history of acute exacerbation, and radiological honeycombing were negative prognostic factors of mortality.

Conclusions: NSIP/UIP pattern of indeterminate for UIP was the major pattern in RA-ILD. Although classifications of HRCT patterns were not related to survival, the presence of radiological honeycombing could be a useful predictor of poor prognosis, and acute exacerbation of ILD can seriously impact patient survival regardless of the presence of a UIP or indeterminate for UIP pattern. Our modified HRCT classification based on the latest IPF guideline might be useful to assess appropriate strategies of diagnosis in future RA-ILD studies, and radiological honeycombing could better predict poor prognosis rather than HRCT pattern.

Keywords: Honeycombing; interstitial lung disease (ILD); prognosis; radiological pattern; rheumatoid arthritis (RA)

Submitted Jun 01, 2019. Accepted for publication Nov 20, 2019.

doi: $10.21037 /$ jtd.2019.11.73

View this article at: http://dx.doi.org/10.21037/jtd.2019.11.73 


\section{Introduction}

Interstitial lung disease (ILD) is one of the most important extra-articular manifestations in patients with rheumatoid arthritis (RA). RA-ILD is associated with high morbidity and mortality $(1,2)$. The presence of a usual interstitial pneumonia (UIP) pattern on radiological and/or histological analysis was shown to be a poor prognostic factor (3-5). Thus, distinguishing the UIP pattern from other patterns in RA-ILD may provide important clinical information on decision-making for treatment and prognosis. Most RA patients have received anti-RA drugs to treat the arthritis itself, and this may have already modified ILD activity (6). Moreover, thoracic surgical procedures in patients with RA-ILD might result in high risk of in-hospital death (7). Therefore, histological analysis should be not routinely performed in most RA-ILD patients, and we think that radiological analysis is particularly important.

In previous reports, radiological analysis, especially by high-resolution computed tomography (HRCT), is used for classification of patterns in patients with RA-ILD. These HRCT patterns are classified primarily as UIP, nonspecific interstitial pneumonia (NSIP), or organizing pneumonia (OP) pattern based on the statement of the American Thoracic Society (ATS)/European Respiratory Society (ERS) in 2002 or ATS/ERS/Japanese Respiratory Society (JRS)/Latin American Thoracic Association (ALAT) in $2013(8,9)$. CT can help in accurately identifying UIP patterns indicating a poor prognosis in RA-ILD (10). However, an overlapping or indeterminate pattern (i.e., unclassifiable pattern other than UIP, NSIP, and OP) is present to some extent (6-52\%) in the RA-ILD population because connective tissue disease (CTD) including RA-ILD presents a diversity of patterns $(4,11)$. Although most studies report UIP and NSIP as being the most common patterns, coexistence of UIP and NSIP is common in RA-ILD $(12,13)$. This might result in varied prediction of mortality. In fact, the 2018 ATS/ERS/JRS/ALAT clinical practice guideline for idiopathic pulmonary fibrosis (IPF) proposed the radiological category of indeterminate for UIP, which might be included in a category consistent with a non-IPF diagnosis such as hypersensitivity pneumonia or CTDILD, with clinicians applying a level of confidence based on the extent of the inconsistent features (14). Therefore, the aim of the present study was to clarify predictive factors of mortality by using HRCT pattern classification based on the recent guideline.

\section{Methods}

\section{Study sample}

This study was approved by the institutional review board of Saitama Red Cross Hospital (approval no. 18-AE). We retrospectively surveyed chronic ILD patients who were positive for anticyclic citrullinated peptide (antiCCP) antibody at our hospital between January 2012 and December 2017 (Figure 1). A diagnosis of RA was made in these patients according to the American College of Rheumatology/European League Against Rheumatism Criteria by rheumatology specialists (15). We then collected data from each patient's medical record that included characteristics, laboratory data, pulmonary function, and chest CT findings at the time of ILD diagnosis. Among the patients with anti-CCP antibody-positive ILD, 7 patients with CTD other than RA, such as microscopic polyangiitis $(\mathrm{N}=1)$, mixed CTD $(\mathrm{N}=2)$, polymyositis/dermatomyositis $(\mathrm{N}=3)$, and psoriatic arthritis $(\mathrm{N}=1)$, were excluded. In addition, 9 patients with anti-CCP-positive ILD who did not develop RA during the follow-up period were also excluded. The remaining patients who were classified as having RA-ILD were also included in this study.

\section{Data collection}

Baseline clinical measures were obtained within 3 months of the initial diagnosis of ILD. Each subject's radiological findings were reviewed by two expert pulmonologists ( $\mathrm{S}$ Sato and H Matsushima) who were blinded to the subject's clinical data. First, patients were classified as presenting a HRCT pattern of definite UIP, probable UIP (Figure $2 A, B$ ), indeterminate for UIP, or alternative diagnosis (Figure $2 C, D, E, F, G, H, I)$ according to the recent guideline (14). In this study, the presence of co-existing small airway disorders such as mosaic attenuation and centrilobular nodules was ignored because a high prevalence of airway abnormalities has been observed in the RA population $(16,17)$. Second, we created a modified HRCT classification as follows. The alternative diagnosis was classified as NSIP, NSIP + OP, $\mathrm{OP}$, and unclassifiable according to previous reports (18). Predominant basilar ground-glass opacity (GGO) with limited to no reticulation and absence of honeycombing were consistent with NSIP (Figure 2D,E,F) (18). NSIP + OP was identified when consolidations were superimposed on a background of GGO with or without reticulations or 


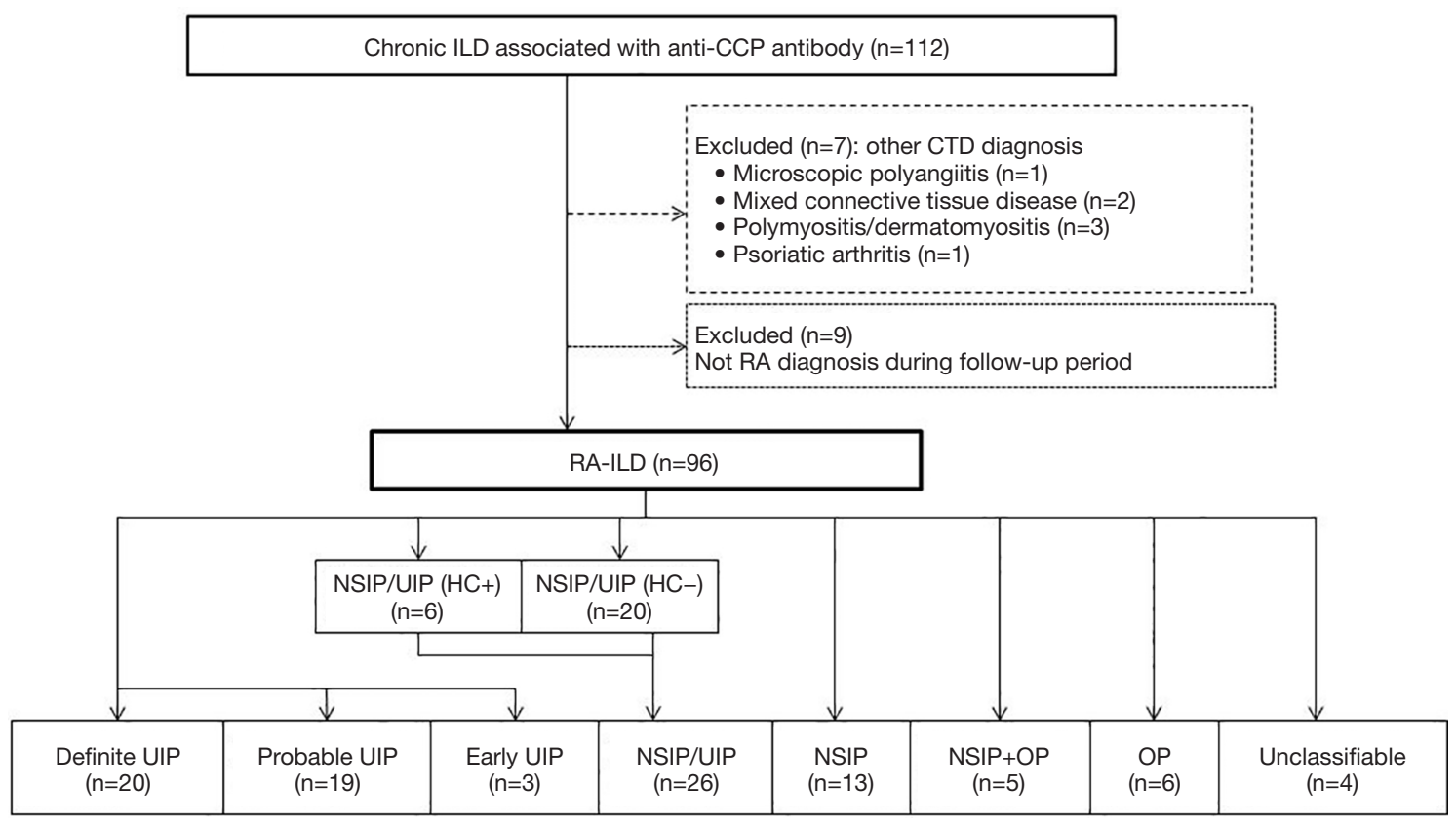

Figure 1 Flow diagram of the patients with RA-ILD. CTD, connective tissue disease; ILD, interstitial lung disease; HC, honeycombing; NSIP, nonspecific interstitial pneumonia; OP, organizing pneumonia; RA, rheumatoid arthritis; UIP, usual interstitial pneumonia.

traction bronchiectasis (18). Moreover, indeterminate for UIP was also classified as early UIP (Figure $2 C$ ) and NSIP/ UIP (Figure 2G,H,I) according to the extent of GGO or distribution of abnormal shadows (i.e., reticulations and GGO) (Figure 3). For example, even if GGO was inconspicuous, both central distribution of reticulation with traction bronchiectasis and subpleural reticulations with or without honeycombing in the lower lung were comparable with NSIP/UIP (Figure 2H,I). For combined pulmonary fibrosis with emphysema (CPFE), positive findings of emphysema were visually defined as presence of a low attenuation indicating the lack of a distinct alveolar wall threshold over $10 \%$ (19). Honeycombing is defined as clustered cystic air spaces with typically comparable diameters of $3-10 \mathrm{~mm}$ in subpleural and lower lobes with well-defined walls (14).

Disagreements between the two pulmonologists after the first assessment were resolved by discussion. Acute exacerbation (AE) was defined based on a previous study, which required all five of the following criteria be met: (I) previous diagnosis of RA-ILD; (II) unexplained worsening of dyspnoea within 30 days; (III) new bilateral GGOs and/or consolidations; (IV) no evidence of pulmonary infection on negative respiratory culture, and (V) exclusion of alternative causes such as left heart failure, pulmonary embolism, or identifiable cause of lung injury (20).

\section{Statistical methods}

The follow-up period was calculated from the date of initial ILD diagnosis until the last visit or time of death. To detect differences between groups, Fisher's exact test or one-way analysis of variance (ANOVA) was used as appropriate. We investigated potential risk factors of mortality with each variable chosen for entry into univariate Cox regression analysis and performed multivariate Cox regression analysis with forward variable selection. The Kaplan-Meier method and log-rank test were used to display and compare survival curves for the cohort stratified for each group. We considered $\mathrm{P}<0.05$ to indicate statistical significance. All data were analysed with SPSS version 22.0 (IBM Japan, Tokyo, Japan).

\section{Results}

\section{Overall patient characteristics}

We identified 96 patients with RA-ILD. They were predominantly female $(61 \%)$ and never-smokers $(53 \%)$ with a mean age of 69 years at the diagnosis of ILD (Table 1). 

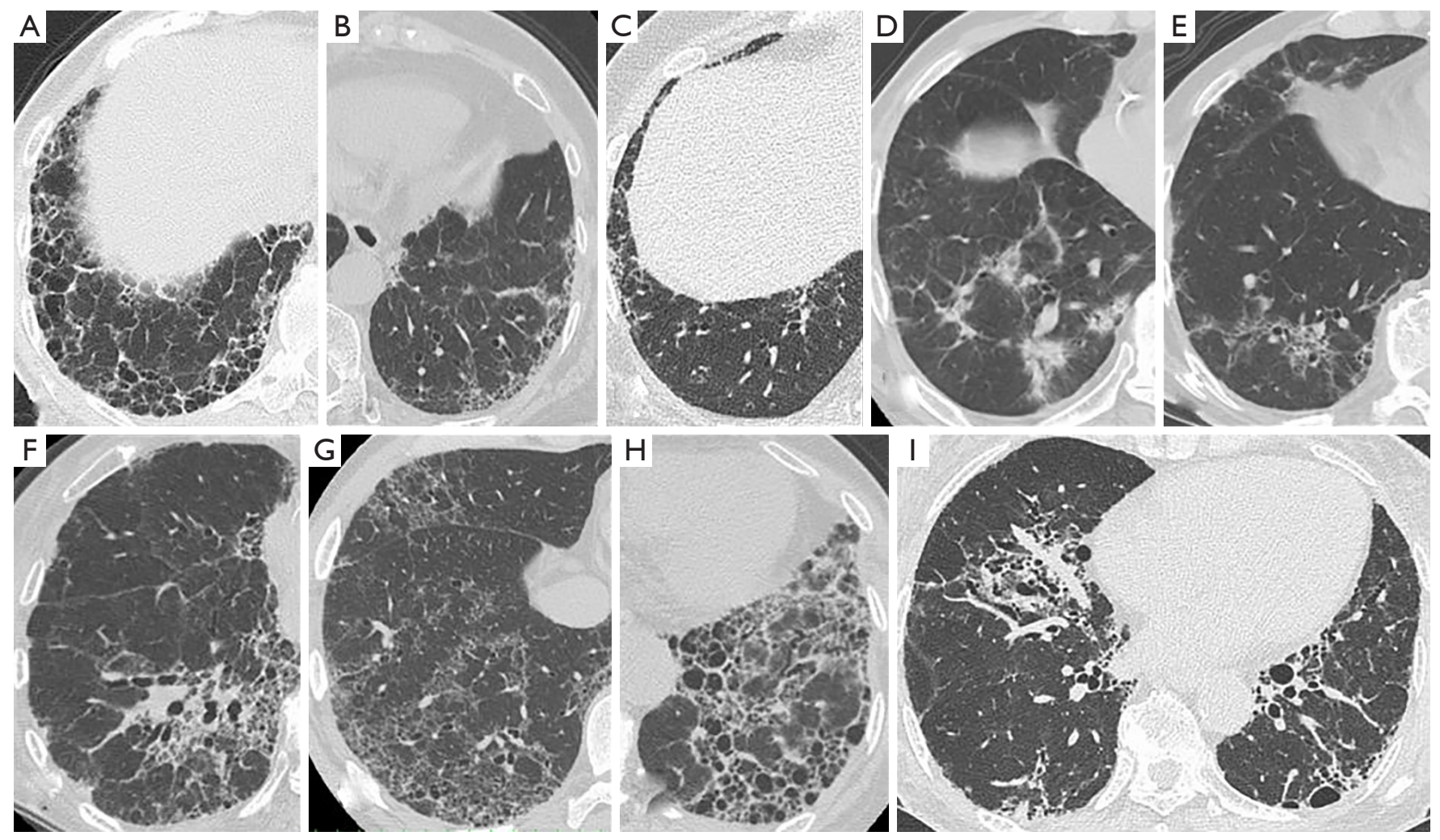

Figure 2 High-resolution computed tomography images illustrating each pattern in RA-ILD. (A) Definite UIP pattern: subpleural and basal-predominant reticulation with clustered cystic space as honeycombing; (B) probable UIP pattern: subpleural and basal-predominant reticulation with peripheral traction bronchiectasis or bronchiolectasis; (C) early UIP pattern: mild reticulation or GGO without traction bronchiolectasis; (D,E,F) NSIP pattern: predominant basilar GGO with limited to no reticulation and absent honeycombing; (G,H,I) NSIP/UIP pattern: both central or diffuse distribution of reticulation or GGO as the component of NSIP and subpleural reticulations with or without honeycombing as the component of UIP in the lower lung (G: diffuse GGO and peripheral reticulation without honeycombing; (H,I): both central and peripheral distribution of reticulation and traction bronchiectasis with honeycombing). GGO, ground-glass opacity; ILD, interstitial lung disease; NSIP, nonspecific interstitial pneumonia; RA, rheumatoid arthritis; UIP, usual interstitial pneumonia.

\begin{tabular}{|c|c|c|c|c|c|}
\hline \multirow{6}{*}{$\begin{array}{l}\text { Lower lung } \\
\text { predominant } \\
\text { distribution }\end{array}$} & \multirow{3}{*}{$\begin{array}{l}\text { Abnormal shadow (GGO or } \\
\text { reticulation) }\end{array}$} & \multicolumn{3}{|c|}{ Subpleural findings (+) } & \multirow{3}{*}{$\begin{array}{l}\text { Subpleural } \\
\text { findings (-) }\end{array}$} \\
\hline & & \multirow{2}{*}{ Honeycombing (+) } & \multicolumn{2}{|c|}{ Subpleural reticulation } & \\
\hline & & & with TB & without TB & \\
\hline & $\begin{array}{l}\text { GGO < extent of reticulation } \\
\text { and peripheral distribution }\end{array}$ & Definite UIP & Probable UIP & $\begin{array}{l}\text { Indeterminate for } \\
\text { UIP [early UIP] }\end{array}$ & - \\
\hline & \multirow{2}{*}{$\begin{array}{c}\text { GGO > extent of reticulation } \\
\text { and/or central or diffuse } \\
\text { distribution }\end{array}$} & $\begin{array}{l}\text { Indeterminate } \\
\text { for UIP NSIP/UIP } \\
\text { (honeycombing +) }\end{array}$ & $\begin{array}{l}\text { Indeterminate } \\
\text { for UIP NSIP/UIP } \\
\text { (honeycombing -) }\end{array}$ & \multirow{2}{*}{\multicolumn{2}{|c|}{ NSIP }} \\
\hline & & \multicolumn{2}{|c|}{ NSIP/UIP } & & \\
\hline
\end{tabular}

Figure 3 Modified computed tomography definitions for definite UIP, probable UIP, indeterminate for UIP, and NSIP. UIP, usual interstitial pneumonia; NSIP, nonspecific interstitial pneumonia; TB, traction bronchiectasis or bronchiolectasis; GGO, ground-glass opacity. 
Table 1 Characteristics of RA-ILD

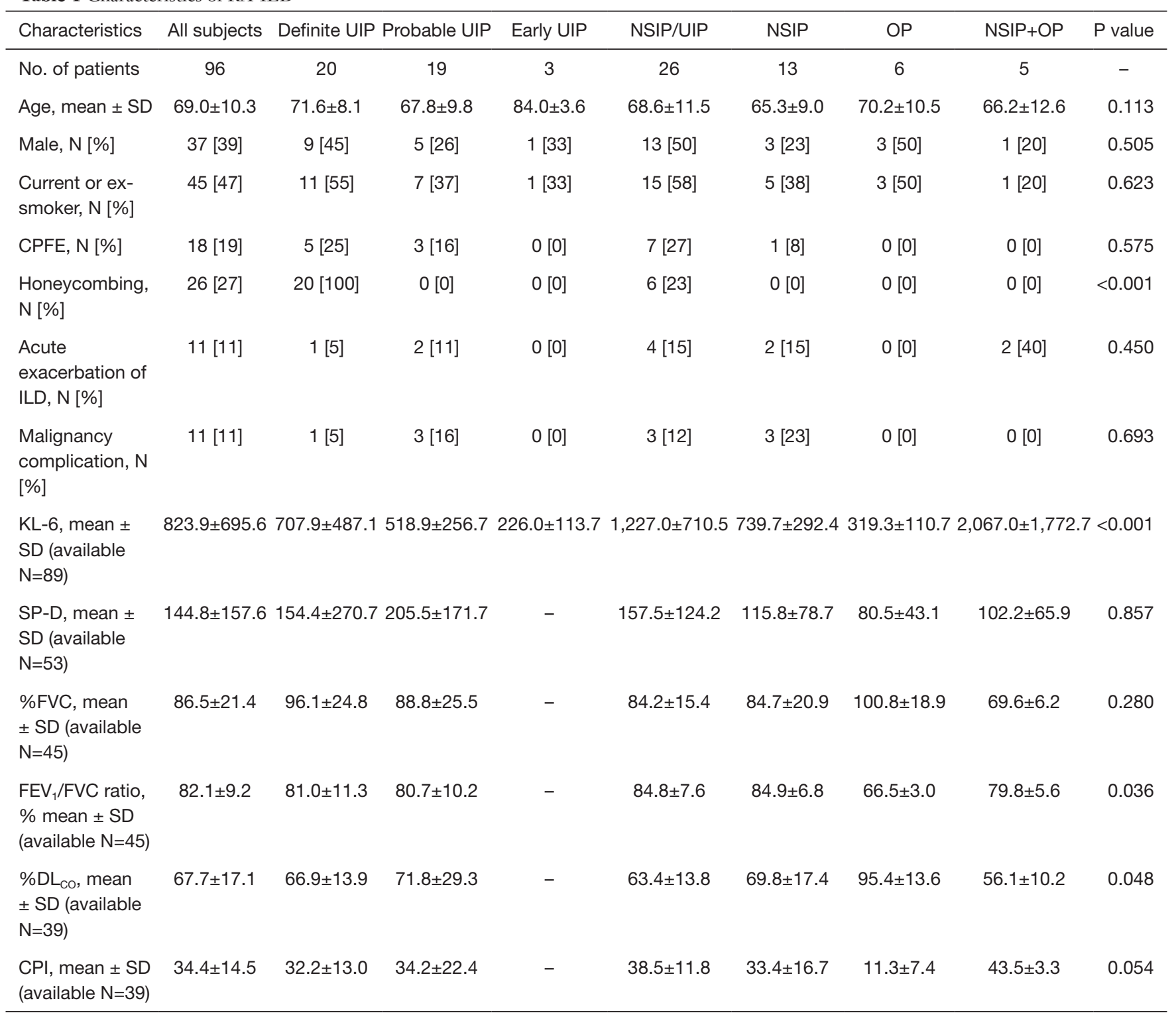

RA, rheumatoid arthritis; ILD, interstitial lung disease; UIP, usual interstitial pneumonia; NSIP, nonspecific interstitial pneumonia; OP, organizing pneumonia; CPFE, combined pulmonary fibrosis with emphysema, KL-6; Krebs von den Lungen-6, SP-D; surfactant protein-D; FVC, forced vital capacity; $\mathrm{FEV}_{1}$, forced expiratory volume in 1 second; CPI; composite physiological index; $\mathrm{DL}_{\mathrm{co}}$, diffusing capacity of the lung for carbon monoxide; SD, standard deviation.

During follow-up, 46 patients (48\%) had ILD preceded by RA (median follow-up: 3.5 years; range, $0.9-42.9$ years), whereas 16 patients $(17 \%)$ with anti-CCP-positive ILD developed RA (median follow-up: 3.9 years; range, 1.2-10.3 years). Thirty-four patients (35\%) were simultaneously diagnosed as having both RA and ILD within 6 months.

A radiologically definite UIP pattern was present in $21 \%(\mathrm{~N}=20)$ of the RA-ILD patients (Figure 1). Other radiological patterns of ILD included probable UIP $(20 \%, \mathrm{~N}=19)$, indeterminate for UIP $(30 \%, \mathrm{~N}=29)$, and alternative diagnosis $(29 \%, \mathrm{~N}=28)$ according to the recent IPF guideline. Our modified HRCT classification included patients with early UIP $(3 \%, \mathrm{~N}=3)$ and NSIP/UIP (27\%, $\mathrm{N}=26$ ), of which some patients were indeterminate for UIP. The alternative diagnosis category included patients with $\operatorname{NSIP}(14 \%, \mathrm{~N}=13), \mathrm{OP}(6 \%, \mathrm{~N}=6), \mathrm{NSIP}+\mathrm{OP}(5 \%)$, 
Table 2 Medications and causes of death in patients with RA-ILD

\begin{tabular}{|c|c|c|c|c|c|c|c|c|c|}
\hline Variables & All subjects & Definite UIP & Probable UIP & Early UIP & NSIP/UIP & NSIP & OP & $\mathrm{NSIP}+\mathrm{OP}$ & $P$ value \\
\hline \multicolumn{10}{|c|}{ Medications (during follow-up), N [\%] } \\
\hline $\begin{array}{l}\text { Corticosteroid } \\
\text { use }\end{array}$ & $50[52]$ & $8[40]$ & $6[32]$ & $1[33]$ & 17 [65] & $8[62]$ & $6[100]$ & $3[60]$ & 0.034 \\
\hline Iguratimod use & $7[7]$ & $0[0]$ & $4[21]$ & $0[0]$ & $2[8]$ & $0[0]$ & $0[0]$ & $0[0]$ & 0.227 \\
\hline $\begin{array}{l}\text { Calcineurin } \\
\text { inhibitor use }\end{array}$ & $30[31]$ & $8[40]$ & $4[21]$ & $0[0]$ & $13[50]$ & $1[8]$ & $1[17]$ & $3[60]$ & 0.044 \\
\hline Biologics use & 19 [20] & $4[20]$ & $4[21]$ & $1[33]$ & $5[19]$ & $2[15]$ & $1[17]$ & $1[20]$ & 0.992 \\
\hline $\begin{array}{l}\text { Acute } \\
\text { exacerbation of } \\
\text { ILD }\end{array}$ & 5 & 1 & 1 & 0 & 3 & 0 & 0 & 0 & \\
\hline $\begin{array}{l}\text { Chronic disease } \\
\text { progression }\end{array}$ & 6 & 2 & 0 & 0 & 2 & 1 & 0 & 1 & \\
\hline Infection & 5 & 2 & 0 & 0 & 1 & 1 & 1 & 0 & \\
\hline Malignancy & 4 & 0 & 1 & 0 & 1 & 1 & 0 & 0 & \\
\hline
\end{tabular}

RA, rheumatoid arthritis; ILD, interstitial lung disease; UIP, usual interstitial pneumonia; NSIP, nonspecific interstitial pneumonia; OP, organizing pneumonia.

and unclassifiable disease $(4 \%, \mathrm{~N}=4)$. Of the patients with NSIP/UIP, 6 had honeycombing and 20 did not. Observer agreement ( $\kappa$ value) for each of HRCT pattern, honeycombing, and CPFE were 0.75, 0.79, and 0.69, respectively.

Serum Krebs von den Lungen-6 (KL-6) was significantly higher in the patients with NSIP + OP but lower in the patients with OP and early UIP (Table 1). Pulmonary function testing showed the forced expiratory volume in 1 second/forced vital capacity (FVC) ratio to be significantly lower in the patients with OP and NSIP+OP, whereas diffusing capacity of the lung for carbon monoxide (DLCO) (\%, predicted) was significantly higher in the patients with $\mathrm{OP}$.

As medication used for RA itself or RA-ILD during follow-up, prednisolone was used in all OP patients, but it tended to be less frequently used in UIP such as definite, probable, and early UIP $(\mathrm{P}=0.034)$ (Table 2$)$. Methotrexate was more frequently used in probable UIP (53\%), and less frequently used in definite UIP (5\%) and NSIP/UIP (12\%) $(\mathrm{P}=0.005)$. Calcineurin inhibitors such as cyclosporine or tacrolimus were more frequently used in the patients with 

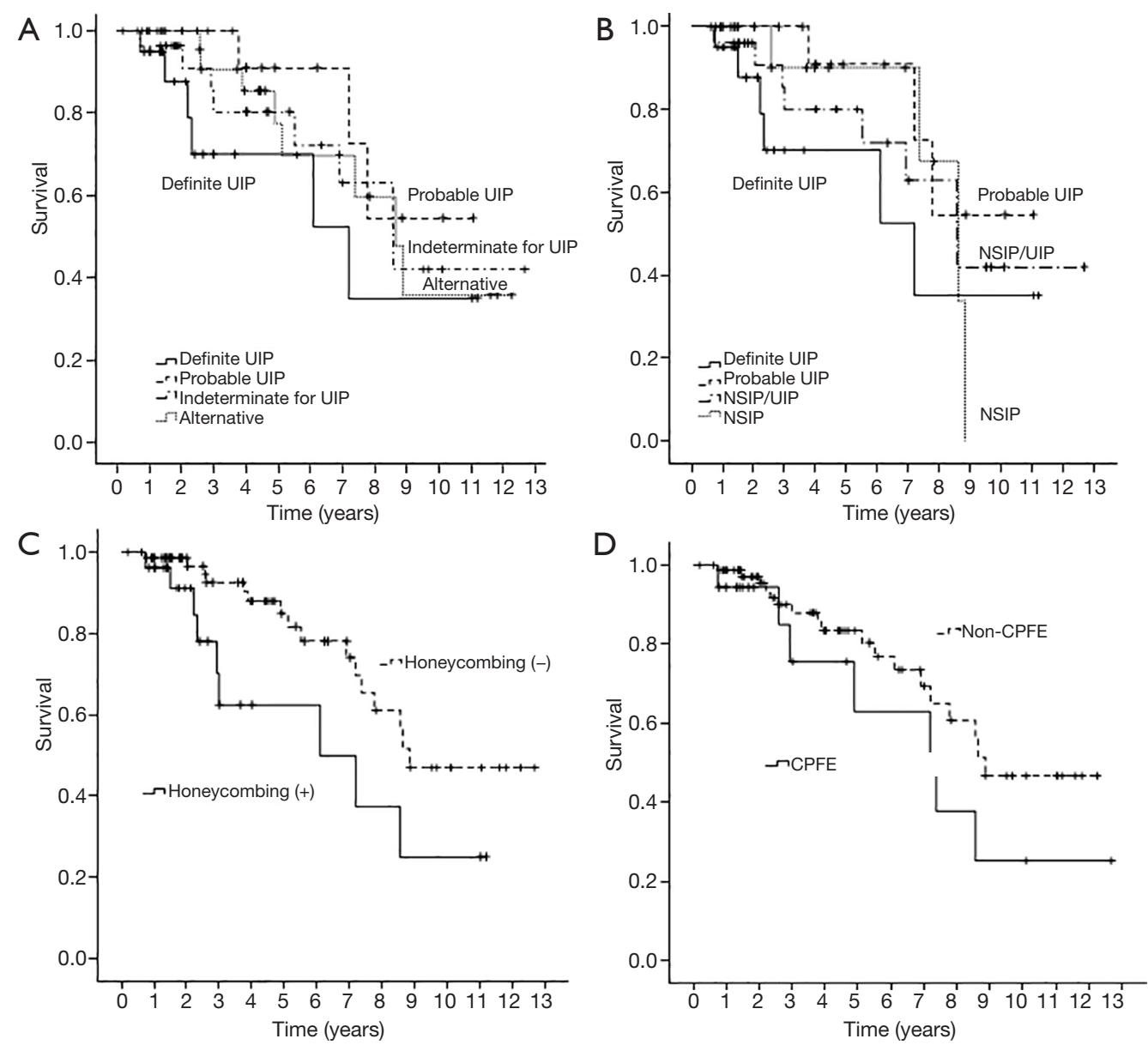

Figure 4 Kaplan-Meier survival curves of all-cause mortality. (A) There were no significant differences in survival between patients with definite UIP, probable UIP, indeterminate for UIP, and alternative diagnosis ( $\mathrm{P}=0.397)$; (B) there were no significant differences in survival between patients with definite UIP, probable UIP, NSIP/UIP, and NSIP ( $\mathrm{P}=0.447$ ). Estimated median survival for definite UIP (6.8 years) did not differ significantly from that for probable UIP (9.1 years, $\mathrm{P}=0.106$ ), NSIP/UIP (8.6 years, $\mathrm{P}=0.430$ ), or NSIP (7.8 years, $\mathrm{P}=0.482$ ); (C) the patients with radiological honeycombing showed poorer survival than those without $(\mathrm{P}=0.024)$; (D) patients with CPFE showed no significant difference in survival compared with those without $\mathrm{CPFE}(\mathrm{P}=0.174)$. CPFE, combined pulmonary fibrosis with emphysema; NSIP, nonspecific interstitial pneumonia; UIP, usual interstitial pneumonia.

definite UIP and NSIP/UIP ( $\mathrm{P}=0.044)$.

\section{Survival}

Death from any cause occurred in 25 patients (26\%) over a median follow-up period of 3.7 years. There were no significant differences in survival between the patients with definite UIP, probable UIP, indeterminate for UIP, and alternative diagnosis ( $\log$ rank $\mathrm{P}=0.397$ ) (Figure $4 A$ ). In our modified HRCT classification analysed for 4 groups because there were too few subjects in other groups, there was no difference in survival between patients with definite UIP, probable UIP, NSIP/UIP, and NSIP (log rank $\mathrm{P}=0.447$ ) (Figure $4 B$ ). Cumulative 5-year survival rates were $70.2 \%$ in definite UIP, $90.9 \%$ in probable UIP, $80.0 \%$ in NSIP/ UIP, and $90.0 \%$ in NSIP patients, respectively. Median survival for patients with definite UIP was 6.8 years [95\% confidence interval (CI): 4.4 to 9.2 ]; with probable UIP, 9.1 years (95\% CI: 7.4 to 10.8 ); with NSIP/UIP, 8.6 years (95\% CI: 6.6 to 10.7 ); and with NSIP, 7.8 years (95\% CI: 6.4 to 9.2 ). Patients with positive findings of radiological honeycombing had significantly worse survival than those without honeycombing ( $\log$ rank $\mathrm{P}=0.024)$ (Figure $4 C$ ). However, survival of patients with CPFE was not 
Table 3 Analyses of predictors of mortality in patients with RA-ILD

\begin{tabular}{|c|c|c|c|c|}
\hline Characteristics & \multicolumn{2}{|c|}{ Univariate Cox regression } & \multicolumn{2}{|c|}{ Multivariate Cox regression } \\
\hline Age & $1.061(1.008-1.117)$ & $0.023^{*}$ & $1.081(1.002-1.165)$ & $0.043^{*}$ \\
\hline Male & $1.203(0.545-2.652)$ & 0.647 & - & - \\
\hline Ex/current smoker & $1.449(0.650-3.229)$ & 0.365 & - & - \\
\hline CPFE & $1.819(0.758-4.365)$ & 0.180 & - & - \\
\hline Honeycombing & 2.499 (1.098-5.692) & $0.029^{*}$ & $3.691(1.053-12.936)$ & $0.041^{*}$ \\
\hline Malignancy complication & $0.786(0.268-2.304)$ & 0.660 & - & - \\
\hline \multicolumn{5}{|l|}{ HRCT pattern } \\
\hline NSIP/UIP & $0.594(0.205-1.722)$ & 0.337 & - & - \\
\hline NSIP & $0.611(0.071-5.285)$ & 0.655 & - & - \\
\hline$\% F V C$ & $1.005(0.977-1.034)$ & 0.734 & - & - \\
\hline$\% \mathrm{DL}_{\mathrm{co}}$ & $0.982(0.947-1.018)$ & 0.323 & - & - \\
\hline $\mathrm{CPI}$ & $1.021(0.979-1.065)$ & 0.328 & - & - \\
\hline $\mathrm{KL}-6$ & $1.000(0.999-1.001)$ & 0.972 & - & - \\
\hline SP-D & $1.003(1.000-1.006)$ & $0.019^{*}$ & Not estimated & - \\
\hline
\end{tabular}

*, $\mathrm{P}<0.05$. RA, rheumatoid arthritis; ILD, interstitial lung disease; HR, hazard ratio; Cl, confidence interval; CPFE, combined pulmonary fibrosis with emphysema; HRCT, high-resolution computed tomography; UIP, usual interstitial pneumonia; NSIP, nonspecific interstitial pneumonia; FVC, forced vital capacity; $\mathrm{DL}_{\mathrm{co}}$, diffusing capacity of the lung for carbon monoxide; $\mathrm{CPI}$, composite physiological index; $\mathrm{KL}$ 6; Krebs von den Lungen-6, SP-D; surfactant protein-D.

significantly different from that of patients without $\mathrm{CPFE}$ $(\log \operatorname{rank} \mathrm{P}=0.174)$ (Figure $4 D)$.

Older age, history of AE, radiological honeycombing, and higher level of serum surfactant protein-D were significant predictors of mortality by univariate analysis. Multivariate Cox proportional hazards analysis showed older age [hazard ration (HR) 1.081, 95\% CI: 1.002 to 1.165], history of AE (HR 6.487, 95\% CI: 1.979 to 21.264), and radiological honeycombing (HR 3.691, 95\% CI: 1.053 to 12.936$)$ to be negative prognostic factors of mortality (Table 3). No relationship was found between the use of medication and mortality in the present cohort of RA-ILD patients.

Causes of death are summarised in Table 2. They included AE $(\mathrm{N}=5)$, chronic disease progression $(\mathrm{N}=6)$, infection $(\mathrm{N}=5)$, cerebro-cardiovascular disease $(\mathrm{N}=4)$, malignancy $(\mathrm{N}=4)$, and unknown $(\mathrm{N}=1)$.

\section{Discussion}

We examined our modified HRCT classification based on the current guideline for IPF as prognostic markers in RAILD patients. The main point of this definition is as follows. The presence of co-existing small airway disorders such as mosaic attenuation and centrilobular nodules was ignored according to previous studies $(16,17)$. In the recent IPF guideline, indeterminate for UIP has features of fibrosis but does not meet the criteria for definite or probable UIP and does not explicitly suggest an alternative diagnosis (14). Considering these two points, we thought that the existence of mild GGO as a HRCT feature or central/ diffuse distribution as the predominant distribution in addition to subpleural and basal predominant reticulation as UIP, should be classified as indeterminate for UIP. Then, by our modified HRCT definition, most patients 
with indeterminate for UIP were classified as having NSIP/UIP. We also found that older age, a history of AE, and radiological honeycombing were significantly poor prognostic factors in the survival analysis. The present study revealed three important points.

First, indeterminate for UIP as a NSIP/UIP pattern was the major HRCT pattern in RA-ILD, and the rate of concordance of HRCT patterns between the two pulmonologists was good. Some previous studies showed that the overall prognosis of patients with a HRCT UIP pattern was significantly worse compared with that in patients with a non-UIP or NSIP pattern $(5,20,21)$. Therefore, in the clinical setting, distinguishing UIP from non-UIP is important to appropriately manage patients with RA-ILD (22). Furthermore, because HRCT is highly predictive of the histological UIP pattern with high specificity, this imaging technique may be a more pragmatic, non-invasive approach for assessing the prognosis of RA-ILD $(5,22)$. However, because lymphoid hyperplasia, peribronchiolar lesion, and mixed UIP and NSIP are frequent pathological findings $(12,13)$, we often cannot classify a HRCT pattern specifically as either UIP or NSIP in clinical practice. Similarly, over a quarter of our RA-ILD subjects presented a NSIP/UIP pattern. This result resembled that of a previous study by Kim et al. (4). Subjects with a NSIP/UIP pattern in the present study might have been forcibly diagnosed as having NSIP or UIP in previous reports. Thus, we considered the possibility that the correlation between HRCT pattern and prognosis could vary. In addition, our modified HRCT classification based on the current IPF guideline showed a high rate of concordance for observer agreement, even though our study might have a biased radiological analysis due to its singlecenter nature. Therefore, this definition might be useful in future RA-ILD studies.

Second, HRCT pattern was not associated with worse survival, but radiological honeycombing predicted a significantly poor prognosis. A recent study showed definite UIP to have a poorer prognosis than probable UIP in RAILD (16). However, Kim et al. reported no difference in median survival between subjects with definite UIP and those with indeterminate NSIP/UIP (4). It is unclear whether radiological honeycombing is linked to prognosis in RA-ILD. As a caveat, we speculate that in previous studies, if patients had radiological honeycombing, they most probably had definite UIP rather than other patterns. Adegunsoye et al. recently noted that honeycombing represents a progressive fibrotic ILD phenotype regardless of underlying disease such as RA, IPF, or chronic hypersensitive pneumonia (23). Therefore, we highlighted honeycombing as the most important radiological finding in clinical practice regardless of a UIP or indeterminate for UIP pattern.

The present study also showed that NSIP did not have a significantly better prognosis than NSIP/UIP, probable UIP, or definite UIP. For example, the median survival times were 6.8 years for definite UIP and 7.8 years for NSIP. This survival time for UIP was longer than previously reported in patients with RA-ILD $(4,5,20,21)$, suggesting that most of our study patients had a mild grade of ILD because about half of them had ILD preceded by a RA diagnosis; in other words, many subjects without respiratory symptoms were diagnosed as having RA-ILD by rheumatologists. From the long-term viewpoint, some patients with idiopathic NSIP had a poor prognosis because of combined malignancy and therapy-related complications such as infections, as noted in our previous study (24). Although we cannot say for certain, the patients with RA-ILD also had similar problems of idiopathic NSIP and thus might not have a significant differently prognosis between NSIP and other patterns.

Third, AE of ILD was the most important factor affecting poor survival in RA-ILD. AE of ILD was involved in $22 \%$ of the causes of death in our cohort. Moreover, $80 \%$ of the patients with UIP or NSIP/UIP and a history of $\mathrm{AE}$ of ILD could not recover and died, although 2 patients with NSIP and a history of AE did recover. Hozumi et al. reported that $\mathrm{AE}$ seriously impacts patient survival, and the a radiological UIP pattern was associated with the development of AE (20). Therefore, if the component of UIP (i.e., subpleural reticulation in the lower lobe) is found to a certain degree, we should recognize the risk of $\mathrm{AE}$ of ILD regardless of a pattern of UIP or indeterminate for UIP (i.e., NSIP/UIP).

The limitations of this study are as follows. First, it was a retrospective, single-center study. Second, selection bias may be present because this study examined only anti-CCP antibody-positive patients with RA-ILD. Although antiCCP is a specific antibody present in a large number of patients with RA (25), our results might not be generalizable to the overall RA population because some patients with RA were negative for anti-CCP antibody. Third, we could not determine whether the clinical diagnosis of RA-ILD impacted treatment decisions and as such, the natural disease course. Fourth, expert thoracic radiologists should analyse HRCT findings, not pulmonologists. Although it is common for pulmonologists to perform HRCT analysis in 
clinical practice, whether analysis by thoracic radiologists is preferable will require further study. Fifth, although we could not quantitatively assess the radiological findings, which would be an issue for future investigation, we believe our modified HRCT classification may offer a useful radiological approach in clinical practice because of the high rate of concordance between the pulmonologists.

In conclusion, our study identified indeterminate for UIP as the major NSIP/UIP pattern in RA-ILD according to our modified HRCT classification referring to the current IPF guideline. The presence of radiological honeycombing could be a useful predictor of poor prognosis. Furthermore, $\mathrm{AE}$ of ILD seriously impacts patient survival regardless of whether a UIP or indeterminate for UIP pattern is present. Use of our modified HRCT classification according to the latest guideline on IPF might aid in the assessment of appropriate strategies of diagnosis in future RA-ILD studies.

\section{Acknowledgments}

We sincerely thank Yosuke Sasaki of Satista Co., Ltd. for his advice on statistical analysis. The authors also thank Rise Japan LLC for the professional English language review. All work was performed at the Saitama Red Cross Hospital.

\section{Footnote}

Conflicts of Interest: The authors have no conflicts of interest to declare.

Ethical Statement: The authors are accountable for all aspects of the work in ensuring that questions related to the accuracy or integrity of any part of the work are appropriately investigated and resolved. This study was approved by the institutional review board of Saitama Red Cross Hospital (approval no. 18-AE).

\section{References}

1. Bongartz T, Nannini C, Medina-Velasquez YF, et al. Incidence and mortality of interstitial lung disease in rheumatoid arthritis: a population-based study. Arthritis Rheum 2010;62:1583-91.

2. Prete M, Racanelli V, Digiglio L, et al. Extra-articular manifestations of rheumatoid arthritis: An update.

Autoimmun Rev 2011;11:123-31.

3. Park JH, Kim DS, Park IN, et al. Prognosis of fibrotic interstitial pneumonia: idiopathic versus collagen vascular disease-related subtypes. Am J Respir Crit Care Med 2007;175:705-11.

4. Kim EJ, Elicker BM, Maldonado F, et al. Usual interstitial pneumonia in rheumatoid arthritis-associated interstitial lung disease. Eur Respir J 2010;35:1322-8.

5. Assayag D, Lubin M, Lee JS, et al. Predictors of mortality in rheumatoid arthritis-related interstitial lung disease. Respirology 2014;19:493-500.

6. Kurata I, Tsuboi H, Terasaki M, et al. effect of biological disease-modifying anti-rheumatic drugs on airway and interstitial lung disease in patients with rheumatoid arthritis. Intern Med 2019;58:1703-12.

7. Caples SM, Utz JP, Allen MS, et al. Thoracic surgical procedures in patients with rheumatoid arthritis. J Rheumatol 2004;31:2136-41.

8. American Thoracic Society; European Respiratory Society. American Thoracic Society/European Respiratory Society International Multidisciplinary Consensus Classification of the Idiopathic Interstitial Pneumonias. This joint statement of the American Thoracic Society (ATS), and the European Respiratory Society (ERS) was adopted by the ATS board of directors, June 2001 and by the ERS Executive Committee, June 2001. Am J Respir Crit Care Med 2002;165:277-304.

9. Travis WD, Costabel U, Hansell DM, et al. ATS/ERS Committee on Idiopathic Interstitial Pneumonias. An official American Thoracic Society/European Respiratory Society statement: Update of the international multidisciplinary classification of the idiopathic interstitial pneumonias. Am J Respir Crit Care Med 2013;188:733-48.

10. Assayag D, Elicker BM, Urbania TH, et al. Rheumatoid arthritis-associated interstitial lung disease: radiologic identification of usual interstitial pneumonia pattern. Radiology 2014;270:583-8.

11. Kelly CA, Saravanan V, Nisar M, et al. British Rheumatoid Interstitial Lung (BRILL) Network. Rheumatoid arthritisrelated interstitial lung disease: associations, prognostic factors and physiological and radiological characteristics-a large multicentre UK study. Rheumatology (Oxford) 2014;53:1676-82.

12. Smith $M$, Dalurzo M, Panse P, et al. Usual interstitial pneumonia-pattern fibrosis in surgical lung biopsies. Clinical, radiological and histopathological clues to aetiology. J Clin Pathol 2013;66:896-903.

13. Cottin V, Hirani NA, Hotchkin DL, et al. Presentation, diagnosis and clinical course of the spectrum of 
progressive-fibrosing interstitial lung diseases. Eur Respir Rev 2018. doi: 10.1183/16000617.0076-2018.

14. Raghu G, Remy-Jardin M, Myers JL, et al. American Thoracic Society, European Respiratory Society, Japanese Respiratory Society, and Latin American Thoracic Society. Diagnosis of Idiopathic Pulmonary Fibrosis. An Official ATS/ERS/JRS/ALAT Clinical Practice Guideline. Am J Respir Crit Care Med 2018;198:e44-68.

15. Aletaha D, Neogi T, Silman AJ, et al. 2010 rheumatoid arthritis classification criteria: an American College of Rheumatology/European League Against Rheumatism collaborative initiative. Ann Rheum Dis 2010;69:1580-8.

16. Jacob J, Hirani N, van Moorsel CHM, et al. Predicting outcomes in rheumatoid arthritis related interstitial lung disease. Eur Respir J 2019. doi: 10.1183/13993003.00869-2018.

17. Perez T, Remy-Jardin M, Cortet B. Airways involvement in rheumatoid arthritis: clinical, functional, and HRCT findings. Am J Respir Crit Care Med 1998;157:1658-65.

18. Zamora AC, Hoskote SS, Abascal-Bolado B, et al. Clinical features and outcomes of interstitial lung disease in antiJo-1 positive antisynthetase syndrome. Respir Med 2016;118:39-45.

19. Ryerson CJ, Hartman T, Elicker BM, et al. Clinical

Cite this article as: Yamakawa $\mathrm{H}$, Sato S, Tsumiyama E, Nishizawa T, Kawabe R, Oba T, Kamikawa T, Horikoshi M, Akasaka K, Amano M, Kuwano K, Matsushima H. Predictive factors of mortality in rheumatoid arthritis-associated interstitial lung disease analysed by modified HRCT classification of idiopathic pulmonary fibrosis according to the 2018 ATS/ERS/ JRS/ALAT criteria. J Thorac Dis 2019;11(12):5247-5257. doi: 10.21037/jtd.2019.11.73 features and outcomes in combined pulmonary fibrosis and emphysema in idiopathic pulmonary fibrosis. Chest 2013;144:234-40.

20. Hozumi H, Nakamura Y, Johkoh T, et al. Acute exacerbation in rheumatoid arthritis-associated interstitial lung disease: a retrospective case control study. BMJ Open 2013;3:e003132.

21. Tsuchiya Y, Takayanagi N, Sugiura H, et al. Lung diseases directly associated with rheumatoid arthritis and their relationship to outcome. Eur Respir J 2011;37:1411-7.

22. Suda T. Up-to-date information on rheumatoid arthritisassociated interstitial lung disease. Clin Med Insights Circ Respir Pulm Med 2016;9:155-62.

23. Adegunsoye A, Oldham JM, Bellam SK, et al. CT honeycombing identifies a progressive fibrotic phenotype with increased mortality across diverse interstitial lung diseases. Ann Am Thorac Soc 2019;16:580-8.

24. Yamakawa H, Kitamura H, Takemura T, et al. Prognostic factors and disease behaviour of pathologically proven fibrotic non-specific interstitial pneumonia. Respirology 2018;23:1032-40.

25. Chou C, Liao H, Chen Ch, et al. The clinical application of anti-CCP in rheumatoid arthritis and other rheumatic diseases. Biomark Insights 2007;2:165-71. 